\title{
Optimising Video Summaries for Mobile Devices using Visual Attention Modelling
}

\author{
Janko Calic \\ Department of Computer Science \\ University of Bristol \\ Woodland Road, Bristol BS8 1UB, UK \\ janko@cs.bris.ac.uk
}

\author{
Neill Campbell \\ Department of Computer Science \\ University of Bristol \\ Woodland Road, Bristol BS8 1UB, UK \\ campbell@cs.bris.ac.uk
}

\begin{abstract}
In order to represent large video collections in the form of key-frame summary on small screen devices, this paper exploits methodology of the visual attention modelling and rapid serial visual presentation. This approach results in an intuitive layout of efficiently generated video summaries. A robust real-time algorithm for key-frame extraction is presented. The system ranks importance of key-frame regions in the final layout by exploiting visual attention modelling. A final layout is created using an optimisation algorithm based on dynamic programming. Algorithm efficiency and robustness are demonstrated by comparing the results with the manually labelled ground truth.
\end{abstract}

\section{Categories and Subject Descriptors}

H.3.7 [Digital Libraries]: Systems issues; G.2.3 [Discrete Mathematics]: Applications

\section{INTRODUCTION}

In spite of numerous attempts to achieve intuitive interaction with large image and video collections, there is a need for efficient algorithms for presentation of visual content, especially in the domain of mobile technology. The conventional presentation paradigm is demanding and attempts to learn and model the way users link perceived stimuli and their meaning [11]. This work makes a shift towards more user centered summarisation and browsing of large video collections by augmenting interaction rather than learning the way users create related semantics. The main focus of this work is augmented browsing of large video collections on mobile and small-screen devices.

In order to create an effortless and intuitive interaction with the overwhelming extent of information embedded in video archives, we propose a system that exploits the model of visual attention and, using extracted salient information, lays out an optimal presentation of the content on a device with a small size display, whether it is a mobile phone, handheld PC or PDA.

The initial step of key-frame extraction, presented in Section 2, utilises underlying production rules to extract the best visual representative of a shot in an efficient manner [5]. Visual attention modelling [8] is used to estimate the most salient regions of extracted key-frames, as given in Section 3 . The number of salient regions is defined by the desired response time, determined from required speed of rapid serial visual presentation [6]. In Section 4 we present the layout algorithm [1] that generates the final video summary using dynamic programming in order to achieve real-time displaying capability in an optimal way. Finally, the results of the algorithms presented are evaluated in Section 5 by comparing achieved output with a manually labelled ground truth.

\section{KEY-FRAME EXTRACTION}

In order to generate the visual summary, a set of the most representative frames is generated from the analysed video sequence. Initially, video data is subsampled in both space and time to achieve real-time processing capability. Spatial complexity reduction is achieved by representing a $8 \times 8$ block with its average pixel value, generating a lowresolution representation of video frames known as the $D C$ sequence. By doing this, the decoding process is minimized since the DC sequence can be efficiently extracted from an MPEG compressed video stream [16]. In the temporal dimension, key-frame candidates are determined either by uniform sampling every $n^{\text {th }}$ frame or after a cumulative pixelwise prediction error between two adjacent candidate frames reaches a predefined threshold. The latter approach distorts the time in a non-linear fashion and thus loses the notion of real motion required by the camera work classification module. Therefore, a temporal decimation with the constant factor of $n=5$ is applied.

Having generated the low complexity data representation with dimensions $W \times H$, a dense optical flow $\vec{F}(x, y)$ of the DC sequence is estimated efficiently using the Lucas-Kanade image registration technique [10]. In order to apply model fitting of optical flow data to a priori generated camera work models (i.e. zoom, tilt and pan), specific transformations are applied to the optical flow $F^{i}(x, y)$ for each frame $i$, as given in Eq. (1)-(4).

$$
\begin{gathered}
\Phi_{z}^{i}(x, y)=\operatorname{sgn}\left(x-\frac{W}{2}\right) F_{x}^{i}(x, y)+\operatorname{sgn}\left(y-\frac{H}{2}\right) F_{y}^{i}(x, y) \\
M_{z}^{i}(x, y)=\Phi_{z}^{i}(x, y) \cdot \omega(x, y) \\
M_{p}^{i}(x, y)=F_{x}^{i}(x, y) \cdot \omega(x, y)
\end{gathered}
$$


Table 1: Camera work categories and corresponding error threshold values

\begin{tabular}{|c||c|c|c|c|c|c|}
\hline & \multicolumn{2}{|c|}{ zoom } & \multicolumn{2}{c|}{ pan } & \multicolumn{2}{c|}{ tilt } \\
\hline & in & out & left & right & up & down \\
\hline \hline$T h_{c w}$ & $<-1.2$ & $>1.2$ & $<-0.7$ & $>0.7$ & $<-0.8$ & $>0.8$ \\
\hline
\end{tabular}

$$
M_{t}^{i}(x, y)=F_{y}^{i}(x, y) \cdot \omega(x, y)
$$

Weighting coefficients $\omega(x, y)$ favour influence of the optical flow in image boundary regions in order to detect camera work rather than a moving object, typically positioned in the centre of the frame. As shown in Eq. 5, the weighting coefficients are calculated as an inverted ecliptic Gaussian aligned to the frame center, with spatial variances determined empirically as $\sigma_{x}=0.4 \cdot W$ and $\sigma_{y}=0.4 \cdot H$.

$$
\omega(x, y)=1-e^{-\left(\frac{(x-W / 2)^{2}}{\sigma_{x}}+\frac{(y-H / 2)^{2}}{\sigma_{y}}\right)}
$$

The measure of optical flow data fitness for a given camera work model is calculated as a normalised sum of $M_{c w}^{i}(x, y)$ for each type of camera work $(c w)$ : $\operatorname{zoom}(z)$, pan $(p)$ and tilt $(t)$, as given in Eq. 6. If the absolute value of fitness function becomes larger than the empirically predefined threshold $T h_{c w}$, the frame $i$ is labelled with one of the six camera work categories, as given in Table 1.

$$
\Psi_{c w}^{i}=\frac{1}{w h} \sum_{x=1}^{W} \sum_{y=1}^{H} M_{c w}^{i}(x, y), \text { where } c w \in\{z, p, t\}
$$

Finally, the binary labels of camera work classes are denoised using morphological operators retaining the persistent areas with camera motion while removing short or intermittent global motion artefacts.

Once the shot regions are labelled with appropriate camera work, only the regions with a static camera (i.e. no camera work labelled) are taken into account in selection of the most representative key-frame candidates. This approach was adopted after consulting the views of video production professionals as well as inspection of manually labelled ground truth. The conclusions were that: i) since the cameraman tends to focus on the main object of interest using a static camera, the high-level information will be conveyed by the key-frame in regions with no camera work labels, ii) chances to have artefacts like motion and out-offocus blur are minimised in those regions.

Subsequently, frames closest to the centre of mass of the frame candidates' representation in a multidimensional feature space are specifically ranked to generate the list of region representatives. The algorithm for key-frame selection is as follows:

\section{Select $N \geq N_{k f}$ candidates from static regions}

2. Calculate feature matrixes for all candidates

\section{Loop through all candidates}

(a) Rank them by $L_{2}$ distance to all unrepresented frames of the analysed shot in ascending order

(b) Select the first candidate and label its neighbouring frames as represented

(c) Select the last candidate and label its neighbouring frames as represented

\section{Export $N_{k f}$ selected key-frames as a prioritised list}

The feature vector used to represent key-frame candidates is a $18 \times 3 \times 3 \mathrm{HSV}$ colour histogram, extracted from the DC sequence representation for reasons of algorithm efficiency. As an output, the algorithm returns a sorted list of $N_{k f}=8$ frames and the first frame in the list is used as the keyframe in the final video summary. In addition to the single key-frame representation, this algorithm generates a video skim for each shot in the video sequence ${ }^{1}$. By alternately selecting the first and the last frame from the ranked list, a balance between the best representability and discovery of unanticipated content is achieved.

\section{VISUAL ATTENTION MODEL}

Having extracted key-frames from video data, salient image regions are determined in order to optimise available display space and show the most important image parts. In order to achieve this a model of bottom-up salient region selection is employed [7]. This salient region selection algorithm estimates the approximate extent of attended visual objects and simulates the deployment of spatial attention in a biologically realistic model of object recognition in the cortex [15]. In our case, this model determines the visual attention path for a given key-frame and automatically selects regions that can be visually attended in a limited time interval.

This approach follows the idea of Rapid Serial Visual Presentation (RSVP), a technique that displays visual information using a limited space in which each piece of information is displayed briefly in sequential order [12]. The RSVP method proved to be especially interesting for video summarisation [14]. We adopt the RSVP method that generates a spatial layout of presented content together with the temporal sequencing. The proposed technique combines the timing of the RSVP with the reaction time of the visual attention model to generate easily readable spatial layout of presented content in a novel and efficient way.

Initially, a set of early visual features, comprising normalised maps of multi-scale center-surround differences in colour, intensity and orientation space, is extracted for each key frame, as presented in [8]. A winner-take-all (WTA) neural network scans the saliency map for the most salient location and returns the location's coordinates. Finally, inhibition of return is applied to a disc-shaped region of fixed radius around the attended location in the saliency map. Further iterations of the WTA network generate a cascade of successively attended locations in order of decreasing saliency.

Knowing the cascade of attended regions and reaction time needed to attend them, a predefined parameter $\mathcal{T}_{\max }$ selects a set of $N$ most important salient regions $R_{i}, i=$ $1, \ldots, N$ if $\mathcal{T}_{N}<\mathcal{T}_{\max }$. In other words, we select the salient regions that can be attended in a fixed time interval $\mathcal{T}_{\text {max }}$. Afterwards, a Gaussian distribution is fitted to a union set of the saliency regions $\mathbb{R}=\bigcup_{i=1}^{N} R_{i}$, as given in Eq. 7 .

$$
\Gamma_{j}(x, y)=e^{-\left(\left(\frac{x-\mu_{x j}}{\sigma_{x j}}\right)^{2}+\left(\frac{y-\mu_{y j}}{\sigma_{y j}}\right)^{2}\right)}
$$

${ }^{1}$ Depending on application type, length of the skim can be either predefined ( $N_{k f}=$ const.) or adaptive, driven by the number of static camera regions and maximum distance allowed during the ranking process 


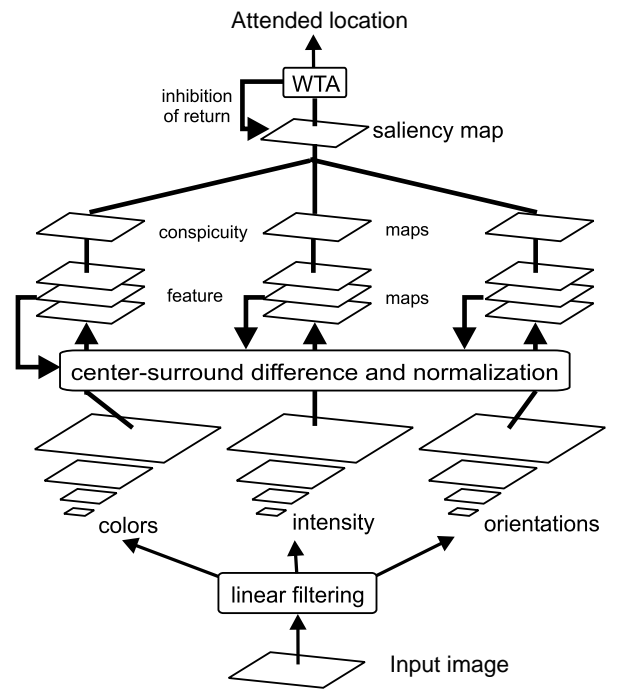

Figure 1: Processing scheme of the model of saliency-based visual attention

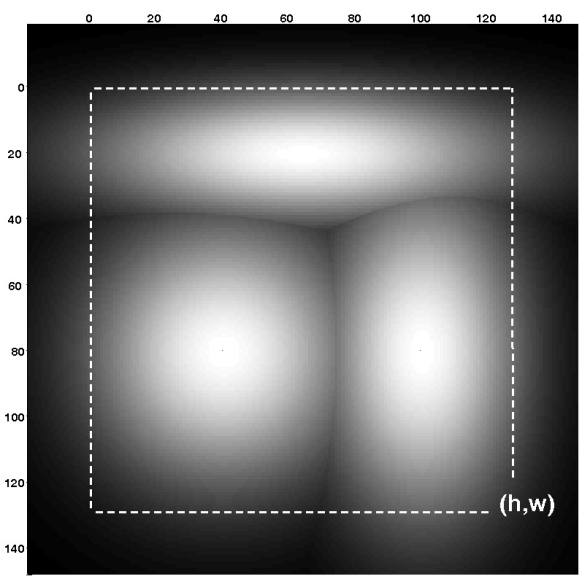

Figure 2: An example of three Gaussians fit into a display size $(h, w)$

The Gaussian parameters $\left\langle\mu_{x j}, \sigma_{x j}, \mu_{y j}, \sigma_{y j}\right\rangle$ are determined for each key-frame $j$ defining the location and size of their most important parts. This information is later utilised in the layout algorithm. The RSVP timing is calculated as a sum of time intervals $\mathcal{T}_{\max }$ for all key-frames in the layout.

\section{LAYOUT ALGORITHM}

After determining the Gaussian parameters $\left\langle\mu_{x j}, \sigma_{x j}, \mu_{y j}\right.$, $\left.\sigma_{y j}\right\rangle$ of the most relevant image region for each key-frame $j$, the objective is to lay out selected salient image parts in an optimal way for a given display size. An example of three salient image regions modelled by Gaussian functions fitted into a display with size $(h, w)$ is depicted in Fig. 2 .

There have been numerous attempts to solve the problem of discrete optimisation for spatio-temporal resources [9]. In our case, we need to utilise the available two-dimensional space given the sizes of salient image regions. However, unlike many well studied problems like stock cutting or bin packing [13], there is a requirement to fit the salient image regions into a predefined area in a given order. In addition, the majority of proposed algorithms are based on heuristics and do not offer an optimal solution.

Therefore, we propose an optimal solution using dynamic programming that is a modification of our algorithm presented in [2]. Dynamic programming finds an optimal solution to an optimisation problem $\min \varepsilon\left(x_{1}, x_{2}, \ldots, x_{n}\right)$ when not all variables in the evaluation function are interrelated simultaneously:

$$
\varepsilon=\varepsilon_{1}\left(x_{1}, x_{2}\right)+\varepsilon_{2}\left(x_{2}, x_{3}\right)+\ldots+\varepsilon_{n-1}\left(x_{n-1}, x_{n}\right)
$$

In this case, solution to the problem can be found as an iterative optimisation defined in Eq. 9 and Eq. 10, with initialisation $f_{0}\left(x_{1}\right)=0$.

$$
\begin{gathered}
\min \varepsilon\left(x_{1}, x_{2}, \ldots, x_{n}\right)=\min f_{n-1}\left(x_{n}\right) \\
f_{j-1}\left(x_{j}\right)=\min \left[f_{j-2}\left(x_{j-1}\right)+\varepsilon_{j-1}\left(x_{j-1}, x_{j}\right)\right]
\end{gathered}
$$

By adopting this algorithm we claim that optimisation of the overall layout error is equivalent to optimisation of the sum of independent error functions of two adjacent images $x_{j-1}$ and $x_{j}$. In our case, the error function is defined as a sum of parts of Gaussians that fell outside of display boundaries $(h, w)$ in a given layout. Knowing the overall sum of Gaussians, given in Eq. 11), and the sum of the parts within the display boundaries, given in Eq. 12, the error function for two adjacent images is defined in Eq. 13.

$$
\begin{gathered}
\gamma_{j}=\sum_{\forall x, y} \sum_{j} \Gamma_{j}(x, y)=\pi \sigma_{x}^{j} \sigma_{y}^{j} \\
\delta_{j}=\sum_{x=1}^{w} \sum_{y=1}^{h} \Gamma_{j}(x, y) \\
\varepsilon_{j-1}\left(x_{j-1}, x_{j}\right)=\gamma_{j}+\gamma_{j-1}-\delta_{j}-\delta_{j-1}
\end{gathered}
$$

The search domain for each pair of Gaussians $\left\{\Gamma_{j}, \Gamma_{j+1}\right\}$ comprises uniformly quantised locations of the secondary Gaussian $\Gamma_{j+1}$ rotated around the primary Gaussian $\Gamma_{j}$. The distance between the centres of $\Gamma_{j}$ and $\Gamma_{j+1}$ is quantised so that the ellipses defined by equations $\Gamma_{j}=$ const. have their semiaxes as follows:

$$
\begin{aligned}
a_{j} & =\sqrt{2} \cdot \mathcal{K} \cdot \sigma_{x} \\
b_{j} & =\sqrt{2} \cdot \mathcal{K} \cdot \sigma_{y} \\
\mathcal{K} & \in\{1,2,3\}
\end{aligned}
$$

Locus of the centre of $\Gamma_{j+1}(x, y)$ relative to the centre of $\Gamma_{j}(x, y)$ is derived from the condition that the two ellipses touch, i.e. their tangents coincide:

$$
\begin{aligned}
x_{r}\left(t_{j}, \mathcal{K}\right) & =a_{j} \cdot \cos \left(t_{j}\right)+a_{j+1} \cdot \cos \left(t_{j+1}\right) \\
y_{r}\left(t_{j}, \mathcal{K}\right) & =b_{j} \cdot \sin \left(t_{j}\right)+b_{j+1} \cdot \sin \left(t_{j+1}\right) \\
t_{j+1} & =\arctan \left(\frac{a_{j} \cdot b_{j+1}}{a_{j+1} \cdot b_{j}} \tan \left(t_{j}\right)\right)
\end{aligned}
$$

The rotation angle $t \in[-3 \pi / 4, \pi / 4]$ is uniformly quantised into 9 values, eliminating the possibility of positioning new salient region above or to the left of the previous one.

The dependency between non-adjacent images is precisely and uniquely defined through the hierarchy of the DP solution tree and there is no limitation of the boundary effect described in detail in [4]. Therefore, the solution to 
the discrete optimisation of layout driven by parameters $\left\langle\mu_{x j}, \sigma_{x j}, \mu_{y j}, \sigma_{y j}\right\rangle$ and the display size $(h, w)$ is practically optimal.

The proposed layout algorithm comprises following procedural steps:

1. Determine Gaussian parameters $\left\langle\mu_{x j}, \sigma_{x j}, \mu_{y j}, \sigma_{y j}\right\rangle$ for all images

2. For each pair of adjacent images:

(a) Determine corresponding cost function values $C(i)$

(b) Form the error function table $\varepsilon_{j-1}\left(x_{j-1}, x_{j}\right)$ as given in Eq. 13

(c) Find optimal $f_{j-1}\left(x_{j}\right)$ and save it

3. If all DP tree branches exploited all available images, roll back through the path with minimal overall cost function $f$

This procedure finds the optimal fit for saliency regions described by a Gaussian with parameters $\left\langle\mu_{x j}, \sigma_{x j}, \mu_{y j}, \sigma_{y j}\right\rangle$. The final step is to determine the rectangular boundaries for image cropping given the optimal fit. This is done by finding the intersection of each pair of Gaussian surfaces $\Gamma_{1}, \Gamma_{2}$ and a plane $\Psi$ through their centre points normal to $x y$ plane, defined by the Eq. 20 .

$$
\Psi: \quad y=\mu_{y 1}+\left(x-\mu_{x 1}\right) \frac{\mu_{y 2}-\mu_{y 1}}{\mu_{x 2}-\mu_{x 1}}
$$

The intersection $\Gamma_{1} \cap \Gamma_{2} \cap \Psi$ is the minimum value on the shortest path between two centres on a surface $\Gamma_{1} \cup \Gamma_{2}$. The optimal cropping is calculated for all $N$ images on the page, generating $N(N-1) / 2$ possible cropping rectangles. The cropping that maximises the value of overall sum within display boundaries $\Omega$, given in Eq. 21, is applied.

$$
\Omega=\sum_{j=1}^{N} \sum_{x=1}^{w} \sum_{y=1}^{h} \Gamma_{j}(x, y)
$$

Finally, the source images are cropped, laid out and displayed on the screen. A number of generated layouts is presented in the following section.

\section{RESULTS}

The experiments were conducted on a large video archive of wildlife rushes, a collection available as a part of the ICBR project [3]. Approximately 12000 hours of digitised footage has been indexed with shot boundary metadata used by the key-frame extraction module. First of all, we present evaluation of the key-frame extraction algorithm, followed by experimental results of the layout algorithm.

\subsection{Evaluation of key-frame extraction}

The evaluation of the key-frame extraction algorithm is undertaken by comparing achieved results to the hand labelled ground truth. Two video clips with approximately 90 minutes of wildlife rushes from the ICBR database were labelled by a production professional, annotating the good $(\mathbb{G})$, bad $(\mathbb{B})$ and excellent $(\mathbb{X})$ regions for a potential location of the key-frame. In order to numerically evaluate the quality of the first version, two precision measures were

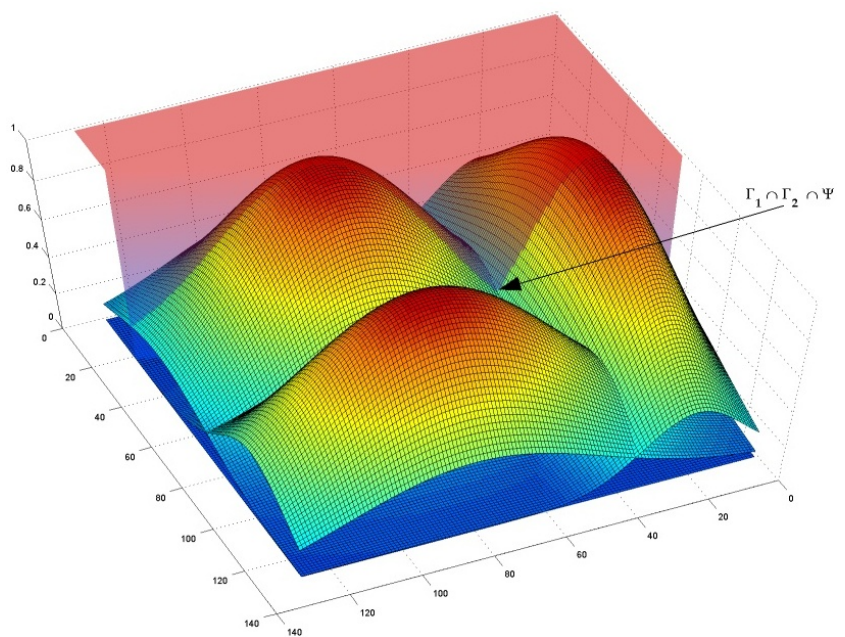

Figure 3: Locating the cropping points at intersection of two Gaussian surfaces $\Gamma_{1}$ and $\Gamma_{2}$ and the $\Psi$ plane defined by two center points $\left(\mu_{1}^{x}, \mu_{1}^{y}\right)$ and $\left(\mu_{2}^{x}, \mu_{2}^{y}\right)$. Thanks to Tilo Burghardt for this figure.

Table 2: Key-frame extraction evaluation results compared to hand labelled ground truth

\begin{tabular}{|c||c|c|c|c|c|cc|}
\hline & $\mathbb{X}$ & $\mathbb{G}$ & $\mathbb{N}$ & $\mathbb{B}$ & $\mathbb{S}$ & $\operatorname{Pr}_{1}[\%]$ & $\operatorname{Pr}_{2}[\%]$ \\
\hline \hline T01000.mov & 52 & 121 & 23 & 14 & 210 & 93.5 & 93.3 \\
T01002.mov & 10 & 129 & 32 & 42 & 213 & 73.6 & 80.3 \\
\hline
\end{tabular}

defined as follows:

$$
\begin{aligned}
\operatorname{Pr}_{1,2} & =D_{1,2} /\left(D_{1,2}+B\right) \\
D_{1} & =2 * X+G-N \\
D_{2} & =X+G+N
\end{aligned}
$$

The value $D_{1}$ incorporates the higher importance of excellent detections and penalise detections that fell into the unlabelled regions $(\mathbb{N})$, while $D_{2}$ takes into account only the fraction of key-frame locations that did not fall within regions labelled as bad. The precision results for the two hand labelled tapes with $\mathbb{S}$ shots are given in Table 2.

\subsection{Layout results}

The results of layout module are presented in Fig. 4 and Fig. 5. The first figure depicts source key-frames with over-
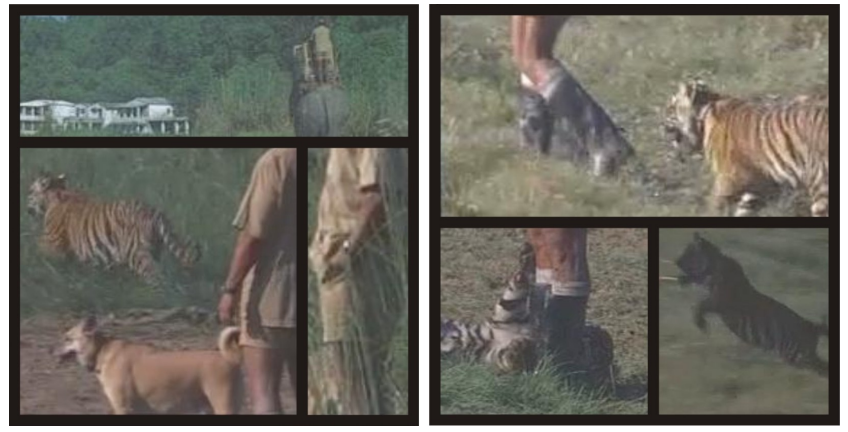

Figure 5: Final screen layouts for cropped keyframes 


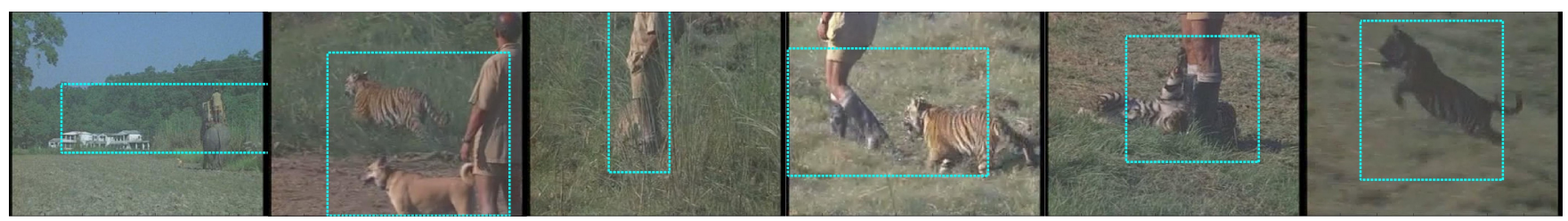

Figure 4: Examples of key-frame cropping using visual attention modelling

layed rectangular cropping regions $\left\langle\mu_{x j} \pm 2 \cdot \sigma_{x j}, \mu_{y j} \pm 2 \cdot \sigma_{y j}\right\rangle$. The maximal time reaction parameter $\mathcal{T}_{\max }$ is empirically set to $300 \mathrm{~ms}$. The results show excellent selection of regions chosen by the attention model, especially knowing that the algorithm has been designed to operate in a fully unsupervised manner.

Two examples of the final layouts are depicted in Fig. 5. One can observe that the layouts do covey major features of the content and its semantics, while maintaining dense packing of the images on a small size screen. This method runs in real-time on a standard PC configuration, allowing for live production of summaries while the video is being played or broadcasted.

\section{CONCLUSION}

This paper presents a video summarisation and browsing algorithm that generates summaries for small screen devices. The algorithm exploits visual attention modelling and creates visual summaries in an efficient and user centered way.

A robust real-time key-frame extraction algorithm exploits production rules to select the best visual representative for a given shot. The results are evaluated by comparing them with the ground truth that was manually labelled by production professionals. The image cropping driven by the visual attention model shows excellent results in conveying semantics as well as appearance of the summarised content. Finally, the layout algorithm that utilises dynamic programming achieves a high density of relevant information displayed on a small size screen.

The future work will be directed towards an extension of the summarisation algorithm towards interactive representation of visual content. Having potential of creating layouts on various types of displays and a fast system response, this algorithm could be used for interactive search and browsing of large video and image collections.

\section{REFERENCES}

[1] R. E. Bellman and S. E. Dreyfus. Applied Dynamic Programming. Princeton University Press, Princeton, NJ, 1962.

[2] J. Ćalić and N. Campbell. Comic-like layout of video summaries. In Proc. of the rth Workshop on Image Analysis for Multimedia Interactive Services (WIAMIS 2006), 2006.

[3] J. Ćalić, N. Campbell, A. Calway, M. Mirmehdi, T. Burghardt, S. Hannuna, C. Kong, S. Porter, N. Canagarajah, and D. Bull. Towards intelligent content based retrieval of wildlife videos. In Proceedings of the 6th International Workshop on Image Analysis for Multimedia Interactive Services (WIAMIS 2005), April 2005.
[4] J. Ćalić, D. Gibson, and N. Campbell. Efficient layout of comic-like video summaries. IEEE Transactions on Circuits and Systems for Video Technology (under review), 2006.

[5] J. Ćalić and B. Thomas. Spatial analysis in key-frame extraction using video segmentation. In Workshop on Image Analysis for Multimedia Interactive Services, April 2004.

[6] O. de Bruijn and R. Spence. Rapid serial visual presentation: a space-time trade-off in information presentation. In AVI '00: Proceedings of the working conference on Advanced visual interfaces, pages 189-192, New York, NY, USA, 2000. ACM Press.

[7] L. Itti and C. Koch. Computational modelling of visual attention. Nat Rev Neurosci, 2(3):194-203, March 2001.

[8] L. Itti, C. Koch, and E. Niebur. A model of saliency-based visual attention for rapid scene analysis. IEEE Transactions on Pattern Analysis and Machine Intelligence, 20(11):1254-1259, 1998.

[9] A. Lodi, S. Martello, and M. Monaci. Two-dimensional packing problems: A survey. European Journal of Operational Research, 141(2):241-252, 2002.

[10] B. D. Lucas and T. Kanade. An iterative image registration technique with an application to stereo vision. In Proceedings of the rth International Joint Conference on Artificial Intelligence (IJCAI '81), pages 674-679, April 1981.

[11] A. W. M. Smeulders, M. Worring, S. Santini, A. Gupta, and R. Jain. Content-based image retrieval at the end of the early years. IEEE Trans. Pattern Anal. Mach. Intell., 22(12):1349-1380, 2000.

[12] R. Spence. Rapid, serial and visual: a presentation technique with potential. Information Visualization, 1(1):13-19, 2002.

[13] P. E. Sweeney and E. R. Paternoster. Cutting and packing problems: a categorized, application-oriented research bibliography. J. Operational Research Society, 43(7):691 - 706, 1992.

[14] T. Tse, G. Marchionini, W. Ding, L. Slaughter, and A. Komlodi. Dynamic key frame presentation techniques for augmenting video browsing. In AVI '98: Proceedings of the working conference on Advanced visual interfaces, pages 185-194, New York, NY, USA, 1998. ACM Press.

[15] D. Walther. Interactions of visual attention and object recognition: computational modeling, algorithms, and psychophysics. PhD thesis, California Institute of Technology, Pasadena, CA, 2006.

[16] B. L. Yeo and B. Liu. Rapid scene analysis on compressed video. IEEE Transactions on Circuits and Systems for Video Technology, 5(6):533-544, 1995. 